\section{Revista}

Ibero-Americana

de Estratégia

\title{
GOVERNANÇA E IMPLEMENTAÇÃO DA ESTRATÉGIA EM ARRANJOS PRODUTIVOS LOCAIS PARA MELHORAS NO DESEMPENHO
}

\author{
GOVERNANCE AND STRATEGY IMPLEMENTATION IN LOCAL CLUSTERS TO \\ IMPROVE THE PERFORMANCE
}

\section{GOBERNANZA Y IMPLEMENTACIÓN DE LA ESTRATEGIA EN ARREGLOS PRODUCTIVOS LOCALES PARA MEJORAS EN EL DESEMPEÑO}

\section{Helen Fischer Günther}

Mestranda em Administração pela Universidade Federal de Santa Catarina - UFSC

Professora da Universidade Federal de Santa Catarina - UFSC

Email: fghelen@yahoo.com.br (Brasil)

\section{Maurício Fernandes Pereira}

Doutor em Engenharia de Produção pela Universidade Federal de Santa Catarina - UFSC

Professor da Universidade Federal de Santa Catarina- UFSC

Email: mfpcris@gmail.com (Brasil)

\section{Murialdo Loch}

Mestrando em Engenharia e Gestão do Conhecimento pela Universidade Federal de Santa Catarina - UFSC

Email: murialdose@yahoo.com.br (Brasil)

\author{
Alexandre Marino Costa \\ Doutor em Engenharia de Produção pela Universidade Federal de Santa Catarina - UFSC \\ Professor do Departamento de Ciências da Administração da Universidade Federal de Santa \\ Catarina - UFSC \\ Email: marino@cse.ufsc.br (Brasil)
}




\title{
GOVERNANÇA E IMPLEMENTAÇÃO DA ESTRATÉGIA EM ARRANJOS PRODUTIVOS LOCAIS PARA MELHORAS NO DESEMPENHO
}

\section{RESUMO}

As micro, pequenas e médias empresas (MPME) encontram-se direcionadas a atuar cooperativamente, a fim de obter benefícios distintos da ação isolada, o que favorece o desenvolvimento de arranjos produtivos locais (APL). Emerge a necessidade de sinergia entre os atores, por meio de mecanismos de integração, que devem ser geridos para a eficácia. Estas características impactam os objetivos e as estratégias. Aí se encontra um dos desafios da atualidade: como aplicar as estratégias, os planos e, como fazer a estratégia funcionar? A governança surge, então, como um meio para alcançar objetivos e, dessa forma, implica a ampliação da atenção ao desenvolvimento de métodos de gestão para manter as idiossincrasias, na mesma medida em que contribuam para a eficácia e melhora no desempenho dos APL.

Palavras-chave: Implementação da Estratégia; Arranjos Produtivos Locais; Governança; Desempenho.

\section{GOVERNANCE AND STRATEGY IMPLEMENTATION IN LOCAL CLUSTERS TO IMPROVE THE PERFORMANCE}

\begin{abstract}
Micro, small and medium enterprises (MSME) are designed to operate in networks, cooperatively, in order to obtain benefits that would not occur through the alone action, and encourage the development of local productive arrangements (LPA). Emerge the need for synergy between the actors, operationalized by the mechanisms of integration, which must be managed for efficiency. These characteristics of cooperation and interaction impact the achievement of the objectives and strategies, and administrative functions and plans. Therein appear one of the challenges of present time: how to implement the strategies, plans, and how to make the strategy works? Governance emerges, then, as a medium to achieve the objectives and, so, talk about it in the LPA extends the focus on developing management methods to maintain the idiosyncrasies, to the same extent as they contribute to the effectiveness and improvement in performance.
\end{abstract}

Keywords: Strategy Implementation; Local Productive Arrangements; Governance; Performance.

Revista Ibero-Americana de Estratégia - RIAE, São Paulo, v. 9, n. 2, p. 99-114, mai./ago. 2010. 


\section{GOBERNANZA Y IMPLEMENTACIÓN DE LA ESTRATEGIA EN ARREGLOS}

PRODUCTIVOS LOCALES PARA MEJORAS EN EL DESEMPEÑO

\section{RESUMEN}

Las micro, pequeñas y medianas empresas (MPME) se encuentran dirigidas a actuar cooperativamente a fin de obtener beneficios que no se produciría a través de la acción solo, y favorece el desarrollo de arreglos productivos locales (APL). Emerge la necesidad de sinergia entre los actores, a través de mecanismos de integración, que deben ser gestionados por la eficacia. Estas características impactan el logro de los objetivos y las estrategias. Ahí radica uno de los desafíos del actual tiempo: ¿cómo aplicar las estrategias, los planes, y, cómo hacer la estrategia funcionar? La gobernanza surge, entonces, como un medio para alcanzar los objetivos y, de tal manera, hablar de ella en los APL amplía la atención al desarrollo de métodos de gestión para mantener las idiosincrasias, en la misma medida en que contribuyan a la eficacia e mejora en el desempeño.

Palavras-chave: Implementación de la Estrategia; Arreglos Productivos Locales; Gobernanza; Desempeño. 


\section{INTRODUCCIÓN}

La globalización y los cambios tecnológicos están creando nuevas fuentes de competencia, la desregulación del mercado, cambios en las reglas de la competencia y la complejidad creciente de los mercados (Day, Reibstein, 1999). El mundo se ha vuelto altamente conectado y el flujo de información permite a las empresas reaccionar más rápidamente a los competidores.

Así, tanto las grandes empresas forman alianzas estratégicas para reducir la creciente competencia, cuánto las micro, pequeñas y medianas empresas (MPME) actúan para reducir al mínimo sus dificultades de operación, a través de redes de cooperación, que constituye una opción estratégica (Costa, Pinheiro, 2007). Estas organizaciones se encuentran dirigidas a actuar en red, en forma cooperativa, a fin de obtener beneficios que no se produciría a través de la acción solo.

Este contexto favorece la creación y el desarrollo de arreglos productivos locales (APL), que ahora ocupan la agenda de los debates y de la construcción de políticas de desarrollo regional (Aun; Carvalho; Kroeff, 2005).

Al analizar las aglomeraciones observe una serie de objetivos económicos, sociales y políticos que actúan con objetivo común y fuertes lazos de interés. Los APL buscan desarrollar ambientes colectivos de intercambio de la información, del conocimiento y de aprendizaje para promover la cooperación entre los agentes locales (Aun, Carvalho, Kroeff, 2005), para alcanzar los objetivos del grupo y, por lo tanto, contribuir para los objetivos de de cada empresa componente.

Emerge, en esta escena, la necesidad de sinergia entre los actores, operacionalizada por los mecanismos de integración, que deben ser gestionados por la eficacia, siendo crucial la gestión de lo APL. Este proceso de gestión, sin embargo, es complejo, porque los APL reúnen a varios agentes (empresas, agencias gubernamentales, instituciones de investigación y desarrollo, asociaciones, etc.), donde tiene el entrelazamiento entre las circunstancias diversas.

Estas características de cooperación, interacción y complejidad impactan el logro de los objetivos y las estrategias (lo que la organización quiere ser), las funciones administrativas y, en consecuencia, los planes. Estos, a su vez, deben ser aplicados, puestos en práctica para alcanzar los objetivos y las estrategias. Ahí radica uno de los desafíos del actual tiempo: ¿cómo aplicar las estrategias, planes, por último, cómo hacer la estrategia funcionar?

Gobernanza surge, entonces, como un medio para alcanzar los objetivos, actuando a favor de ellos, puede ser entendido como un conjunto de ideas que pueden ir hacia el norte a las actividades humanas - todas las formas de acción colectiva. Es el proceso mediante el cual las sociedades o las organizaciones toman las decisiones importantes, determinan quién debe participar y la forma en

Revista Ibero-Americana de Estratégia - RIAE, São Paulo, v. 9, n. 2, p. 99-114, mai./ago. 2010. 
que son responsables, siendo que las estructuras y la calidad de la gobernanza constituyen factores determinantes de la cohesión o el conflicto social, el éxito o el fracaso del desarrollo económico (Santos, Coelho, Santos, 2005: 288, 290-291).

De tal manera, discutir la gobernanza en los APL amplía la atención a las formas de organización y gestión de estas organizaciones complejas, respectando y, sobre todo, guardar el fortalecimiento de su idiosincrasia. Por lo tanto, desarrollar métodos de gestión para mantener las características de cooperación, comunicación y innovación en la misma medida en que contribuyan a la eficacia y mejora en el desempeño ocasionados por esta acción combinada.

Desde el tema presentado, es pertinente destacar el propósito de este estudio, guiado por el objetivo general de comprender la gobernanza en los arreglos productivo locales (APL) con mira a su contribución a la implementación de la estrategia. Específicamente, el objetivo es analizar la formación e características de los APL, investigar aspectos generales de la gobernanza, y discutir la gobernanza y su contribución a la implementación de la estrategia en los APL.

\section{REVISIÓN LITERARIA}

\subsection{ARREGLOS PRODUCTIVOS LOCALES}

Cocco (2006) presenta el concepto de las agrupaciones locales como el resultado de la "traducción" lingüística y conceptual de Systèmes Productifs Locaux, existentes en Francia y basado en el concepto de National Innovation System, creado en Gran Bretaña. Así, el término "arreglo" se relaciona con los elementos previstos, sino también al azar, una aglomeración determinada (cluster) de las unidades territoriales de la misma cadena de suministro industrial (Cocco, 2006).

El SEBRAE (2003: 12) define APL como grupos de empresas ubicadas en el mismo territorio, que se han especializado de producción y mantener un vínculo de conexión, integración, cooperación y aprendizaje entre sí y con otros actores locales.

Sin embargo, Cassiolato y Lastres (2003: 27) afirman que los APL son aglomeraciones de integración económica regional, política y social, centrándose en un conjunto específico de actividades económicas. Tiene lazos, a pesar de incipientes, con la participación y la interacción de las empresas. También se incluyen varias instituciones públicas y privadas para la formación de recursos humanos (tales como colegios y universidades); investigación, desarrollo e ingeniería; la política, la promoción y financiación.

Revista Ibero-Americana de Estratégia - RIAE, São Paulo, v. 9, n. 2, p. 99-114, mai./ago. 2010. 
Así, la cooperación es una condición necesaria para la configuración de un APL, que el objetivo de los componentes es mejorar la competitividad, porque la cooperación es esencial para la dinámica de la competitividad de los APL (Stainsack, 2006: 9).

Britto (2004) indica que la cooperación es una herramienta eficaz para el tratamiento de la información, una alternativa importante para la agregación de capacidades complementarias, para permitir una mejor hacer frente a las turbulencias y facilitar la identificación y explotación de nuevas oportunidades tecnología.

El autor también señala que la cooperación a menudo objetiva ventajas competitivas relacionadas con la explotación de nuevas oportunidades y, por lo tanto, debe ser capaz de generar resultados concretos adecuados para los participantes del arreglo. Por último, destaca la importancia de mantener la cooperación para facilitar la comunicación entre los agentes, que permitan la integración de sus competencias, la consolidación de los principios de confianza mutua y una mayor sincronización de las acciones y estrategias que han adoptado (Britto, 2004: p. 2-3).

De este bosquejo de la posible cooperación puede identificar los flujos de información entre los interesados y por lo tanto, la característica de la comunicación en el entorno de APL. Por lo tanto, Aun, Carvalho y Kroeff (2005) muestran que los flujos de información entre las empresas es esencial para lograr una ventaja competitiva sostenible. Además, los autores comentan que en el contexto de los APL, debemos considerar los ambientes colectivos para intercambiar información, conocimiento y aprendizaje que fomenten la cooperación entre los actores locales.

Así, el intercambio de información es esencial para mejorar la capacidad de las organizaciones y el aprendizaje. La construcción del conocimiento, cuando en cooperación, tiene los atributos de resultado colectivo de la combinación de diversos elementos, que junto cumplen las necesidades individuales y organizacionales. Así, el conocimiento de un individuo, al interactuar con el conocimiento de otros individuos, resulta en nuevos conocimientos individuales y en algunos dominios colectivos (Tomaél, Marteleto, 2006).

Albagli y Loureiro (2008) caracterizan ambiente informacional mediante la identificación de los principales actores, sus papeles y relaciones, así como otros elementos que definen las condiciones de acceso, necesidades y usos de la información. Aun, Carvalho y Kroeff (2005) ponen os arreglos productivos locales como el ambiente para estudio y análisis de las diferentes formas de realización de las interacciones entre los actores, especialmente con respecto a la creación y el intercambio de conocimientos, que crean condiciones para la innovación.

Los APL son, entonces, un ambiente para la innovación que aporta elementos materiales (empresas, instituciones educativas y organizaciones de investigación, apoyo, infraestructura),

Revista Ibero-Americana de Estratégia - RIAE, São Paulo, v. 9, n. 2, p. 99-114, mai./ago. 2010. 
inmateriales (información, conocimiento, capacidad de aprendizaje) e institucionales (las actitudes de y las normas sociales, las políticas públicas y marco legal), que comprenden una compleja red de relaciones favorables a la innovación (Albagli, 2006:6).

Pinto y Souto (2007) advierten que los estudios sobre las experiencias desarrolladas por medio de los APL deben considerar la cuestión de la innovación como catalizador para el desarrollo económico, ya que este es el objetivo que motiva la aglomeración de las organizaciones.

Sin embargo, Aun, Carvalho y Kroeff (2005) señalan que la innovación y el aprendizaje interactivo son tal vez las fuerzas más importantes de los arreglos productivos. De esto, los autores argumentan que la innovación puede estar inserida en los APL en el fomento de la interacción entre los distintos agentes y asociaciones que fomentan las redes interactivas de aprendizaje, influyen en la producción y la mejora, en particular, la capacidad innovadora de agentes.

La proximidad institucional, normas compartidas, convenciones, valores, expectativas y rutinas que nacen de la experiencia común conformada por las instituciones se consideran importantes para lograr una ventaja competitiva sostenible (Aun; Carvalho; Kroeff, 2005). En este sentido, es el espacio para la gobernanza de los APL, a fin de promover esta aproximación institucional.

Por último, Santos, Coelho y Santos (2005: 293) explican que la construcción de procesos de comunicación y coordinación facilita el proceso de gobernabilidad, para garantizar que las posibilidades tecnológicas encontradas sean validadas mediante la adopción de decisión y transformación en acciones concretas.

\subsection{GOBERNANZA}

Existen diversas formas de la gobernanza, pasando por varios contextos, como las empresas anónimas, el gobierno y, los arreglos productivos. El término gobernanza, en su sentido moderno, era casi desconocido hasta el final del siglo pasado, mientras que las palabras adquieren significado a través del uso en el tiempo, no es de extrañar los actuales debates sobre su significado, que no tiene una definición única, pero sí, varias ideas sobre lo que debería o podría significar en diferentes situaciones (Santos, Coelho, Santos, 2005, p. 287-288).

El término gobernanza se entiende como un conjunto de ideas que pueden ir hacia una dirección a las actividades humanas - todas las formas de acción colectiva (Santos; Coelho; Santos, 2005: 288). Según el SEBRAE (2003: 67): se refiere a las diversas formas en que los individuos y las instituciones (públicas y privadas) administran sus problemas comunes, cuenta los intereses

Revista Ibero-Americana de Estratégia - RIAE, São Paulo, v. 9, n. 2, p. 99-114, mai./ago. 2010. 
divergentes o diferentes y lleva a cabo acciones de cooperación. No sólo afecta a las instituciones formales y los regímenes de coordinación y autoridad, sino también los sistemas informales.

Lodi (2000: 24) se refiere a la gobernanza de las empresas privadas, llamadas de gobernanza corporativa (GC), con el siguiente concepto, tomado del Informe Cadbury: sistema por el cual las empresas son dirigidas y controladas. El Instituto Brasileiro de Governança Corporativa - IBGC, a su vez, indica que la GC es el sistema por el cual se dirige, supervisa y promueve las organizaciones, por medio de las relaciones entre propietarios, administradores, directores y órganos de supervisión. Principios de buena gobernanza de las empresas se traducen en recomendaciones que sean objetivas, equiparando sus intereses con el fin de preservar y aumentar el valor de la organización, facilitando su acceso al capital y contribuyendo a su longevidad ${ }^{1}$.

Susigan, García y Furtado (2002) se refieren al concepto de gobernanza por la coordinación entre todos los actores involucrados, cuya estructura muestra la jerarquía, el liderazgo y el mando, ejercida por las empresas participantes. En consecuencia, el SEBRAE (2003) caracteriza a la gobernanza como los diferentes modos de liderazgo, coordinación, intervención, participación y negociación de conflictos en los procesos de decisiones locales.

Se plantea, entonces, una pregunta: ¿existen diferencias entre la gobernanza y la gestión? ¿Cuáles son las diferencias y similitudes?

Santos, Coelho y Santos (2005: 288) distinguen la gobernanza de la gestión con respecto a cómo las grandes decisiones son tomadas - para lograr objetivos importantes, mantener relaciones clave y proporcionar información. Los autores presentan una definición que incluye estas ideas complejas: la gobernanza es el proceso mediante el cual las sociedades u organizaciones toman decisiones importantes, determinan quién debe participar y cómo se deben rendir cuentas.

Sin embargo, Coral y Cunha (2009) definen la gobernanza como un sistema de gestión, con administración dinámica y democrática, que considera las necesidades de las personas involucradas en el APL, incluye herramientas para la toma de decisiones dentro de los objetivos del arreglo, estimula el intercambio de información y el aprendizaje, fomenta la cooperación, cambiando la lógica de la acción individual.

En una esfera más pragmática de discusión, el APL de Software de Curitiba ${ }^{2}$, en su sitio web, menciona que la gobernanza de un APL se compone de todas las partes interesadas (empresas y organizaciones que apoyan) en la gestión de sus problemas y objetivos como un proceso continuo de desarrollo de negocios, a través de prácticas democráticas de articulación.

Por lo tanto, la buena gobernanza, de acuerdo con IBGC, asegura la equidad, la transparencia, la rendición de cuentas (accountability) y la obediencia a las leyes del país 
Helen Fischer Günther, Maurício Fernandes Pereira, Murialdo Loch \& Alexandre Marino Costa

(compliance). Esto demuestra la importancia de la gobernanza empresarial para la prosperidad de los negocios y la rendición de cuentas (Lodi, 2000: 63).

Por lo tanto, establecer sistemas eficaces de gobernanza debe ser una prioridad para cualquier empresa que quiera competir a nivel mundial. La imagen del Consejo sale como órgano responsable de la formulación de estrategias, seguimiento de los resultados, establecimiento del presupuesto de capital y estructura de financiación, en suma, el proceso de generar y aprovechar el valor de la empresa (Rodrigues; Mendes, 2004: 114).

Una cosa a considerar es que las pequeñas empresas no tienen el costumbre de pensar estratégicamente, en parte por la falta de órganos específicos para la definición de programas y proyectos que resulten en la generación de más valor de negocio (Rodrigues, Mendes, 2004).

Así, Rodrigues y Mendes (2004: 113) atribuyen la gestión estratégica a la Junta con el intento de determinar la dirección estratégica con miras a horizontes a largo plazo, por lo que es el uso de oportunidades, la selección, estudio y ejecución de proyectos de expansión que debe aumentar el valor de la empresa.

\subsection{ESTRATEGIA Y SU IMPLEMENTACIÓN}

En el entorno social actual, económico y de organización, se observa una clara tendencia a volver a los valores de coherencia, sentido común y equilibrio, lo que afecta directamente a la manera de pensar acerca de las estrategias de organización. La búsqueda desenfrenada de beneficios, la formulación del producto que no se adhiera al cliente y la mera imposición de reglas y normas que el cuerpo de organización, son ejemplos de ideas inadecuadas para su examen en una planificación de la organización hoy en día.

La estrategia, según Mintzberg, Lampel y Ahlstrand (2002) puede ser considerada la planificación (dirección, orientación, ruta), (modelo estándar que garantiza la coherencia en el tiempo, teniendo en cuenta las estrategias que desee, las realizadas y las emergentes), la posición (en relación con los clientes y del mercado) la perspectiva (la visión de la empresa internamente y en relación con este medio ambiente) y la trampa (no siempre logra maniobra).

Mintzberg (1998) sugiere una imagen de la creación de artesanía como la que más se parezca el proceso de elaboración de estrategias, porque es un proceso de aprendizaje en lugar de determinar el futuro de la organización. Por lo tanto, es imperativo conocer íntimamente la organización y sus actividades, tanto la forma en que funciona, lo que produce, que se forma, las materias primas, sus capacidades y cómo es el mercado, para evitar la creación de estrategias

Revista Ibero-Americana de Estratégia - RIAE, São Paulo, v. 9, n. 2, p. 99-114, mai./ago. 2010. 
inadecuadas. La estrategia debe tener en cuenta las experiencias pasadas y las perspectivas para el futuro.

Por lo tanto, hay una crítica acerca de la planificación formal y los resultados atribuidos a un solo controlador de la empresa. Mintzberg (1998, p. 422) destaca más los logros de las intenciones (o planes), diciendo que fue un error que el pensamiento es lo primero y la acción, posterior. De hecho, ambos se presentan juntos y se alimentan entre sí, formando la íntima relación entre pensamiento y acción. Este es el secreto de una artesanía y también la creación de una estrategia.

Las estrategias emergentes, por lo tanto, surgen cuando existe la capacidad de aprender y la disponibilidad de recursos, tales como las malas hierbas, y se convierten en la organización cuando se refieren a toda la compañía, orientando la forma en que se comportan. Así, existe la estrategia general, que corresponde a las directrices generales de la empresa (deliberadamente) y los detalles hechos por la otra (emergentes) (Mintzberg, 1998).

Se debe demostrar una inconsistencia en la visión convencional de la gestión estratégica, la de que una estrategia sirve a la estabilidad de la organización, y no para cambiar. En la visión convencional, cualquiera que sea la definición, una estrategia requiere la estabilidad de la organización. La falta de estabilidad significa falta de estrategia (no hay camino para el futuro, ningún modelo del pasado) (Mintzberg, 1998: 428). Por lo tanto, debemos equilibrar la estabilidad y el cambio.

Considerando, pues, las perspectivas actuales en relación con el medio ambiente, el mercado y las organizaciones que conducen a la innovación continua, la flexibilidad y el cambio como algo normal, el capital intelectual debe ser considerado tanto en la formulación, cuanto en la aplicación de estrategias. Por lo tanto, existe la percepción de que la organización puede despertar las energías y el talento de las personas que trabajan allí (TACHIZAWA, CRUZ JR; ROCHA: 128), es repensar las reglas que controlan y se centró en personas y no los resultados, ya que una buena gestión no moldear su comportamiento por las reglas, pero da prioridad al logro de resultados (TACHIZAWA, CRUZ JR \& ROCHA: 188).

Así, se considera que el concepto de organización está ligado a la existencia de objetivos (Etzioni, 1976; Daft, 1999, Perrow, 1981; Katz, Kahn, 1987), y que de esos nacen las estrategias (lo que la empresa quiere ser), las funciones administrativas y, en consecuencia, los planes. Estos, a su vez, deben ser ejecutados, puestos en práctica para alcanzar los objetivos y estrategias. Ahí radica uno de los complejos desafíos de nuestro tiempo: ¿cómo aplicar las estrategias, planes, por último, cómo hacer la estrategia funcionar?

Revista Ibero-Americana de Estratégia - RIAE, São Paulo, v. 9, n. 2, p. 99-114, mai./ago. 2010. 
Hrebiniak (2006) aborda específicamente esta cuestión, señalando que los directivos están capacitados para planificar, en vez de ejecutar y que hacer el trabajo de la estrategia es más difícil que crear la estrategia, debido a la política, la inercia, la resistencia al cambio, el conflicto en la estructura organizativa, deficiencias en el intercambio de información, estrategias y los problemas con la aceptación y la gestión del cambio. Disponer de guías y modelos lógicos y objetivos para la finalización del proceso de ejecución de la estrategia son esenciales para el éxito en la misma medida que la cooperación, comunicación e integración, la interdependencia entre personas, áreas y funciones. Por otra parte, la responsabilidad que cae en la cuenta de la estrategia de implementación debe ser clara y sin ambigüedades.

¿Por qué, entonces, sigue siendo difícil y complejo aplicar la estrategia?

Podría se pensar que durante muchos años se mantuvo la práctica de la separación entre la concepción y ejecución (Helou, 2008) y por lo tanto las estrategias no eran ejecutadas, debido a la brecha creada entre lo que la organización quería y lo que los trabajadores deseaban, verificada por diversos efectos, tales como el absentismo y la rotación de personal.

\section{MÉTODO}

Este estudio implica una discusión teórica sobre la formación de arreglos productivos locales y sus características, y los aspectos generales de gobernanza y estrategia. Por último, hay la discusión entre la gobernanza, la implementación de la estrategia y las características de los APL.

Por lo tanto, hemos realizado una revisión bibliográfica, hemos seleccionado unos cuantos libros, artículos e informes de investigación pertinentes a los objetivos de esta investigación. Estos materiales fueron leídos, discutidos y compilados en representación de los debates y reflexiones planteadas. Por último, se ha estructurado y preparado el texto final del estudio, que se presenta aquí.

\section{RESULTADOS Y CONCLUSIONES}

La red de acción es, hoy en día, común en los negocios, porque las alianzas permiten tanto a ampliar los mercados, cuanto actuar con más fuerza y precisión. La cooperación surge como un medio de supervivencia, en particular para las micro, pequeñas y medianas empresas (MPME), que a veces forman arreglos llamados productivos locales (APL) junto con otras instituciones (gobierno, universidades, asociaciones e institutos de investigación).

Revista Ibero-Americana de Estratégia - RIAE, São Paulo, v. 9, n. 2, p. 99-114, mai./ago. 2010. 
Emerge, en esta escena, la necesidad de la sinergia entre los actores, operado por estos mecanismos de integración, que deben ser gestionados por la eficacia, en que es fundamental la gestión de lo APL. La aplicación de estrategias puede ser considerada un problema importante, teniendo en cuenta el mosaico de los objetivos y necesidades de un APL. Fueran observados APLs ubicados en el sur de Brasil (en los estados de Santa Catarina y Paraná).

De ello se deduce que la gobernanza es un medio para minimizar los desacuerdos, porque es la forma organizada en que los individuos y las instituciones tienen sus problemas comunes gestionados, en cooperación, por medio de los aspectos de liderazgo, coordinación y participación, como se identifica en revisión literaria. Ademais, la gobernanza es también un medio para obtener mejoras en el desempeño, alcanzar los objetivos eficazmente y mantener la acción de de ellos. En particular, la gobernanza apoya y subvenciona la toma de decisiones, ya que presenta una amplia y clara, y con prácticas encaminadas al éxito común de la APL (y no una empresa u otra institución aislada).

Así, se puede desarrollar múltiples formas y prácticas de acción que respeten las condiciones y características de las empresas y la disposición de las que participan, ampliando la atención a nuevas formas de acción, gestión y organización para fortalecer las idiosincrasias, en la misma medida en que contribuyan a la eficacia.

La información y la comunicación desempeñan un papel crucial en la dinámica de gobernanza, porque, rescatando Britto (2004: 2-3), un menudo de la cooperación genera ventajas competitivas objetivas relacionadas con la explotación de nuevas oportunidades y, por lo tanto, debe ser capaz de crear adecuados resultados concretos a el APL, que permite la integración de sus competencias, la consolidación de los principios de alcalde de una confianza mutua y sincronización de las acciones y estrategias que han seguido.

La comunicación supone, como función, significativo papel en la aplicación efectiva de las estrategias a través de la integración entre departamentos y el liderazgo, las comunicaciones entre los procesos de organización y objetivos de rendimiento bien establecido. También es evidente que el problema de la aplicación de lo que vemos hoy en día puede estar relacionado con la coordinación en el rostro de una actitud de silencio de los responsables de que en las organizaciones. También hay que considerar que el intercambio de información a través de la comunicación es esencial para mejorar la capacidad, la innovación y el aprendizaje de las MPME. En este aspecto, las organizaciones han señalado las dificultades en el uso de la información y la comunicación, que la tecnología quiere minimizar. Significa que el impacto directo de la comunicación y de la aplicación con éxito de la estrategia residen en la coherencia entre (1) lo que

Revista Ibero-Americana de Estratégia - RIAE, São Paulo, v. 9, n. 2, p. 99-114, mai./ago. 2010. 
se busca (2) lo que si ejecuta, y (3) lo que se utiliza como medida de control. Existen diferencias entre el 1, 2 y 3, lo que puede explicar, no como el único factor, el fracaso de su implementación.

Por lo tanto, establecer sistemas eficaces de gobernanza debe ser una prioridad para cualquier empresa que quiera competir en el mundo, ayudando a liberar el potencial y la generación de valor de la empresa y su desempeño, que vienen de sus estrategias.

La gobernanza contribuye a llenar la falta de una estrategia de aplicación eficaz, como la inercia, la resistencia al cambio, el conflicto en la estructura, deficiencias de organización en el intercambio de información, y puede ser considerada como un modelo lógico y objetivo, un guía para la finalización del proceso de ejecución de la estrategia.

Así, entre los factores que influyen en el éxito de las iniciativas políticas se suman a correcta identificación de la existencia de grupos de productores, y la comprensión de las características principales de la estructura productiva. Sin embargo, en Brasil, lo que ha visto en los últimos años es la ausencia de una eficaz coordinación de acciones y, en consecuencia, la superposición y la fragmentación de las acciones institucionales, con derroche de recursos y los resultados que distan mucho de espera para estas políticas (IPEA, 2006).

Por esa razón, la gobernanza es fundamental para la evaluación y el control de los resultados deseados en los proyectos de lo APL, cuyo objetivo es promover la integración entre los agentes, en busca de financiación para los proyectos, promover la cooperación, así como estimular la expansión y la creación de empresas (Stainsack, 2006: 12).

El debate sobre la gobernanza en APL se convierte, entonces, importante para el campo de la Ciencia de la Administración, porque mejora la atención a las formas de organización y gestión de organizaciones complejas. Por lo tanto, es necesario desarrollar métodos de gestión para mantener las características de la cooperación, la comunicación y la innovación en la misma medida en que contribuyan a la eficiencia y a la mejora en el desempeño, ocasionados por esta acción combinada.

Se entiende, por último, que las externalidades generadas por una política de gobernanza tienden a ser positivas, ya que elaboran, debaten y reúnen las diferentes percepciones para promover metas comunes, o, incluso en un sentido amplio, desarrollan estrategias comunes para obtener objetivos comunes, y también ayudan a lograr los objetivos y fortalecer las peculiaridades naturales.

\section{NOTAS}

${ }^{1}$ Disponible en: < http://www.ibgc.org.br/Secao.aspx?CodSecao=17>. Acceso en 19 jun. 2009.

${ }^{2}$ Disponible en: <http://www.apl-sw-cwb.org.br/FreeComponent100content1809.jsp>. Acceso en 21 jun. 2009. 


\section{REFERENCIAS}

Aun, M. P; Carvalho, A. M. A.; Kroeff, R. L. (2005). Aprendizagem Coletiva em Arranjos Produtivos Locais: um novo ponto para as políticas públicas de informação. In: V ENLEPICC, Salvador.

Britto, J. (2004). Cooperação e Aprendizado em Arranjos Produtivos Locais: em busca de um Referencial Analítico. Relatório de Atividades do Referencial Conceitual, Metodológico, Analítico e Propositivo - Redesist, UFRJ.

Cassiolato, J. E; Lastres, H. M. M. (2003). O foco em arranjos produtivos e inovativos locais de micro e pequenas empresas. In: Lastres, H. M. M.; Cassiolato, J. E.; Maciel, M. L. Pequena empresa: cooperação e desenvolvimento local. Rio de Janeiro: Relume Dumará.

Cocco, G. (2006) Mobilizar os Territórios Produtivos: para além do capital social, a constituição do comum. In: Silva, G; Cocco, G. Territórios produtivos: oportunidades e desafios para o desenvolvimento local. Rio de Janeiro: DP\&A; Brasília, DF: Sebrae.

Coral, E. Cunha, A. A. (2009). Definições de APL e Governança de APL: a importância dos conceitos para o desenvolvimento e aumento da competitividade. IEL/SC, mar. Disponível em: <http://www2.fiescnet.com.br/web/recursos/VUVSR01EVTJOdz09>. Acesso em 21 jun. 2009.

Costa, W. J. V; Pinheiro, M. M. K. (2007). Redes Sociais e Compartilhamento de Informação e Conhecimento em Aglomerações Produtivas. In: Informação \& Informação, Londrina, v. 12.

Daft, R. L. (1999). Teoria e projeto das organizações. 6ª ed. Rio de Janeiro: LTC, 1999.

Day, G. S; Reibstein, D. J. (1999) A dinâmica da estratégia competitiva. Rio de Janeiro: Campus.

Etzioni, A. Organizações modernas. 5ª ed. São Paulo: Pioneira, 1976.

Helou, J. A. H. A. (2008). O impacto da consultoria externa no desempenho organizacional, na percepção do público interno: um estudo de caso da empresa XYZ. 2008. 129 f. Dissertação (Mestrado em Administração) - Centro Sócio-Econômico, Universidade Federal de Santa Catarina, Florianópolis.

Hrebiniak, L. G. (2006). Fazendo a estratégia funcionar: o caminho para uma execução bemsucedida. Rio Grande do Sul: Bookman.

Instituto Brasileiro De Governança Corporativa - IBGC. (2009) Disponível em: <http://www.ibgc.org.br>. Acesso em 19 jun. 2009.

Revista Ibero-Americana de Estratégia - RIAE, São Paulo, v. 9, n. 2, p. 99-114, mai./ago. 2010. 
Ipea. (2006). Identificação, Mapeamento e Caracterização Estrutural de Arranjos Produtivos Locais no Brasil.

Katz, D; Kahn, R. L. (1987). Psicologia social das organizações. $3^{a}$ ed. São Paulo: Atlas.

Loureiro, I. M. A; Albagli, S. (2008) O papel do ambiente informacional na geração do conhecimento e inovação. In: IX ENANCIB, 2008, São Paulo-SP. IX Encontro Nacional de Pesquisa em Ciência da Informação (ENANCIB).

Mintzberg, H. (1998). A criação artesanal da estratégia. In: MONTGOMERY, C. A; PORTER, Michael E. Estratégia: a busca da vantagem competitiva. Rio de Janeiro: Campus, p. 419-437.

Mintzberg, H; Lampel, J; Ahlstrand, B. (2002). Todas as partes do elefante. In: JULIO, C. A; SALIBI NETO, J. Estratégia e planejamento. São Paulo: Publifolha / Coletânea HSM Management, p. 09-20.

Perrow, C. (1981). Análise organizacional: um enfoque sociológico. São Paulo: Atlas.

Pinto, M. G.; Souto, M. S. M. L. (2007). Arranjos produtivos locais como ferramenta de desenvolvimento econômico e inovação: um diagnóstico sobre a experiência nacional. In: XIV SIMPEP, São Paulo.

Rodrigues, J. A.; Mendes, G. M. (2004). Governança corporativa: estratégia para geração de valor. Rio de Janeiro: Qualimark.

Santos; Coelho; Santos. (2005). Foresight, engajamento social e novos modelos de governança. In: Centro De Gestão E Estudos Estratégicos. Ciência, tecnologia e sociedade: novos modelos de governança. Brasília: CGEE.

Sebrae. (2003). Termos de Referência para atuação do Sistema SEBRAE em arranjos produtivos locais. Brasília: SEBRAE.

Stainsack, C. (2006). Governança em Arranjos Produtivos Locais: Experiências do Paraná, set. Disponível em: <http://www.ielpr.org.br/apl/uploadAddress/GovernanaAPLsSet06\%5B47828\%5D.pdf>. Acesso em: 25 jun. 2009.

Tachizawa, T; Cruz Júnior, J. B; Rocha, J. A. O. (2006). Gestão de Negócios: visões e dimensões empresariais da organização. $3^{a}$ edição. São Paulo: Editora Atlas.

Tomaél, M. I; Marteleto, R. M. (2006). Do compartilhamento da informação ao conhecimento coletivo. In: VII ENANCIB, 2006, Marília-SP. VII Encontro Nacional de Pesquisa em Ciência da Informação (ENANCIB).

Revista Ibero-Americana de Estratégia - RIAE, São Paulo, v. 9, n. 2, p. 99-114, mai./ago. 2010. 
Gobernanza Y Implementación de La Estrategia en Arreglos Productivos Locales para Mejoras en el Desempeño

Recebido: 29/06/2010

Aprovado: 15/09/2010

Revista Ibero-Americana de Estratégia - RIAE, São Paulo, v. 9, n. 2, p. 99-114, mai./ago. 2010. 